\title{
Spatial Domain Resource Sharing for Overlapping Cells in Indoor Environment
}

\author{
Riichi Kudo, ${ }^{1}$ Yasushi Takatori, ${ }^{1}$ Kentaro Nishimori, ${ }^{1}$ Takeo Ichikawa, ${ }^{1}$ \\ Tomoki Murakami, ${ }^{1}$ Masato Mizoguchi, ${ }^{1}$ and Masahiro Morikura ${ }^{2}$ \\ ${ }^{1}$ NTT Network Innovation Laboratories, NTT Corporation, Yokosuka 239-0847, Japan \\ ${ }^{2}$ Graduate School of Informatics, Kyoto University, Kyoto 606-8317, Japan \\ Correspondence should be addressed to Riichi Kudo, kudo.riichi@lab.ntt.co.jp
}

Received 1 July 2009; Accepted 25 December 2009

Academic Editor: Hongxiang Li

Copyright () 2010 Riichi Kudo et al. This is an open access article distributed under the Creative Commons Attribution License, which permits unrestricted use, distribution, and reproduction in any medium, provided the original work is properly cited.

\begin{abstract}
As microcell wireless systems become more widespread, intercell interference among the access points will increase due to the limited frequency resource. In the overlapping cell scenario, radio resources should be shared by multiple cells. Although time and frequency resource sharing has been described in many papers, there is no detailed report on dynamic spatial resource sharing among multiple cells for microcell wireless systems. Thus, we present the effectiveness of spatial resource sharing among two access points. We introduce two scenarios based on the zero forcing method; one is the primary-secondary AP scenario and the other is the cooperative AP scenario. To evaluate the transmission performance of spatial resource sharing, channel matrices are measured in an indoor environment. The simulation results using the measured channel matrices show the potential of spatial resource sharing.
\end{abstract}

\section{Introduction}

The rapid increase in data traffic has led to strong demands for large-capacity transmission systems. Wireless local area networks (WLAN) and cellular systems are two major wireless access systems that are being targeted to achieve even higher data rates. Orthogonal frequency division multiplexing (OFDM) and multiple input multiple output (MIMO) have been recognized as effective ways to attain higher throughput for IEEE 802.11n [1] and 3G Long term evolution (LTE) [2]. Unfortunately, the recent advances in these techniques still lag the demands being made, so bandwidth of each carrier is being increased from one generation to the next $[1,2]$. Another trend in wireless access systems is the rapid increase in the number of wireless devices. The total number of cellular phone subscribers reached 100 million at the end of December in 2007 in Japan and exceeded the number of land phone subscribers [3]. The number of WLAN chip shipments in each year is also increasing and it reached to 200 million in 2008 [4]. It is thought that people will start putting wireless devices on machines in the near future. This changes the usage of wireless access from people to people (P-to-P) or people to machine (P-to-M) communication to machine to machine (M-to-M) communication and a huge growth in this market can be anticipated $[1,2]$.

The above trends bring new challenges for wireless technologies because both the larger bandwidth and the larger number of wireless devices may cause significant shortages in the frequency channels. One example is the ongoing standardization for very high throughput (VHT) wireless LANs in bands below $6 \mathrm{GHz}$, that is, IEEE802.11ac, which is expected to be the next standard after IEEE802.11n. It has been virtually agreed that IEEE802.11ac will double the maximum bandwidth of IEEE802.11n from $40 \mathrm{MHz}$ to $80 \mathrm{MHz}$ to achieve $1 \mathrm{Gbps}$ throughput at the median access control (MAC) service access point (SAP) [5]. Since the microwave frequency band has been already assigned to various radio systems, it is difficult to expand the frequency bands allocated for wireless LANs into the microwave band. Thus, the number of available frequency channels would be half that of IEEE $802.11 \mathrm{n}$ and is more likely that a frequency channel will be used at a nearby basic service set (BSS), which consists of access point (AP) and associated stations (STAs). 
Therefore, how APs and STAs behave in the overlapping cell among BSS is important [6].

In the overlapping cell scenario, radio resources should be shared by multiple cells. There are three resources to be shared, time, frequency, and spatial resources. The first one, time resource sharing is realized by carrier sense multiple access collision avoidance (CSMA/CA) or request to send/clear to send (RTS/CTS) protocols $[7,8]$. It allocates different time durations to each access to avoid inter-cellinterference (ICI). Dynamic resource sharing in the time domain is actualized by making transmission opportunity proportional to traffic load at each cell. The second one, frequency resource sharing, allocates a different frequency band to each cell to prevent intercell interference. The dynamic channel assignment (DCA) scheme was proposed to improve the spectrum efficiency [9]. The DCA concept has been extended to support the heterogeneous multiple radio system scenario and is considered as a type of cognitive radio technology. The drawback, the frequency domain method is the complexity of the resource allocation procedure. In the following, we will focus on the last radio resource, spatial resource, to improve the spectrum efficiency further.

Compared to time and frequency resource sharing, spatial resources sharing research is relatively immature especially for the overlapping cell scenario. MIMO technology which uses spatial signal processing is employed by the latest wireless access standards such as $3 \mathrm{G}$ long term evolution (LTE) [10] and IEEE802.11n [11]. It increases the channel capacity between one transmitter and one receiver by using spatial domain. MIMO technology has been extended to multiuser MIMO (MU-MIMO) to attain high channel capacity even for multiple simple wireless terminals $[12,13]$. Many spatial resource sharing methods for MUMIMO were proposed to take into account various QoS conditions for isolated cells but not for overlapping cells $[14,15]$. On the other hand, ICI suppression using spatial domain technology, for example, beamforming or smart antennas, was extensively studied to shorten the frequency reuse distance $[16,17]$. It improves the spectrum efficiency in total system level and can be interpreted as one approach for spatial resource sharing. Recently, femto-cell technology was developed to actualize umbrella cells where multiple femto-cells are created inside a macrocell by transmission power adjustments [18]. Although these techniques realize spatial resource sharing even in autonomous systems, they are effective only for static resource allocation, and it remains hard to respond to dynamic traffic changes in each cell. The cooperation of multiple APs was proposed to attain the highest spectrum efficiency for a multiple overlapping cell scenario $[19,20]$. In this method, all APs are connected to an access controller which manages all spatial resources using the channel state information (CSI) of all AP- station (STA) links. Unfortunately, this approach cannot be applied to the general case, for example, overlapping cells in a WLAN, where multiple APs are connected to different wired networks. To the best of our knowledge, no detailed report has examined dynamic spatial resource sharing among multiple cells where each cell is connected to a different network. Thus, this paper focuses on this scenario and presents the throughput improvement obtained by spatial resource sharing for overlapping cells. In spatial resource sharing, AP allocates spatial resources to STAs if the spatial resources are not consumed by the other AP. The conventional AP waits for another time duration even when only one spatial stream is used at the overlapped cells. Therefore, spatial resource sharing for overlapped cell enables dynamic spatial resource allocation and further improvements in spectrum efficiency.

In this paper, two scenarios (both based on the zero forcing method) for spatial resource sharing are considered. One is the primary-secondary AP scenario and the other is the cooperative AP scenario. To evaluate the effectiveness of these methods, a channel state information measurement experiment is conducted in a large office and $8 \times 4$ MIMO OFDM channel matrices for 48 subcarriers are obtained using the short and long preambles based on the IEEE802.11a standard. Although the measurement environment is a large room, we consider the two-room scenario by separating two APs with a "virtual" wall. The simulation results confirm the potential of spatial resource sharing.

This paper is organized as follows. Section 2 describes eigenvector transmission and the impact of spatial resource sharing in channel capacity. In Section 3, we introduce the experimental setup and the measurement environment. Section 4 presents simulation results. Section 5 summarizes the paper.

Throughout the paper, superscript $*$ and superscript $H$, denote the complex conjugate, and the Hermitian transposition, respectively. Term $|\mathbf{A}|$ is the determinant of $\mathbf{A}$.

\section{System Model}

We consider a downlink MIMO system for a single AP and STA in the overlapping cell scenario. To evaluate the effectiveness of spatial resource sharing, we compare the two overlapping cell scenario to the single cells scenario using time resource sharing. In this section, we describe the channel conditions and two scenarios of spatial domain resource sharing; the primary-secondary $\mathrm{AP}$ scenario and the cooperative AP scenario. Furthermore, we focus on the CSI error and show the relationship between CSI and ICI.

\subsection{Channel Model for Time Resource Sharing. It is assumed} that the number of APs is two, each hosts $U$ mobile stations (STAs) and each AP and STA have $N$ transmit antennas and $M$ receive antennas, respectively. We denote this as the $N>M$ system. Here, transmission from AP $k, k=1,2$, is considered. The channel state between AP $k$ and STA $i$ (being hosted by AP $k$ ) is represented by channel matrix $\mathbf{H}_{k, i} \in \mathbb{C}^{M \times N}, k=1,2$, and $i=1,2, \ldots, U$. The $L_{k, i} \times 1$ transmit symbol vector for STA $i, \mathbf{x}_{k, i}$, is transmitted using transmission weight $\mathbf{W}_{k, i} \in \mathbb{C}^{N \times L_{k, i}} . L_{k, i}$ is the number of data streams transmitted simultaneously to STA $i$ from AP $k$. The signal vector received at STA $i$ is given as

$$
\mathbf{y}_{k, i}=\mathbf{H}_{k, i} \mathbf{W}_{k, i} \mathbf{x}_{k, i}+\mathbf{n}_{k, i}
$$


where $\mathbf{n}_{k, i} \in \mathbb{C}^{M \times 1}$ is the additive white Gaussian noise vector and the elements of $\mathbf{n}_{k, i}$ are assumed to have a variance, $\sigma^{2}$. In this paper, $\sigma^{2}$ is set to be one for all STAs. Even if every STA has a different noise variance, we can consider the same situation by normalizing the norm of the channel matrix without loss of generality.

2.2. Channel Model for Spatial Resource Sharing. When spatial resource sharing is active, the two APs can transmit in the same time slot. The received signal vectors at STA $i$, which is communicating with AP 1 , and at STA $j$, which is communicating with AP 2, are given as

$$
\begin{gathered}
\mathbf{y}_{1, i}=\mathbf{H}_{1, i} \mathbf{W}_{1, i} \mathbf{x}_{1, i}+\mathbf{H}_{\mathrm{I}, 2, i} \mathbf{W}_{2, j} \mathbf{x}_{2, j}+\mathbf{n}_{1, i} \\
\mathbf{y}_{2, j}=\mathbf{H}_{2, j} \mathbf{W}_{2, j} \mathbf{x}_{2, j}+\mathbf{H}_{\mathrm{I}, 1, j} \mathbf{W}_{1, i} \mathbf{x}_{1, i}+\mathbf{n}_{2, i},
\end{gathered}
$$

where $\mathbf{H}_{\mathrm{I}, l, i} \in \mathbb{C}^{N \times M}$ is the interference channel matrix between AP $l$ and STA $i$ of AP $k$, where $l$ is 1 or 2 and $l \neq k$. Therefore, $\mathbf{H}_{\mathrm{I}, 1, i}$ and $\mathbf{H}_{\mathrm{I}, 2, j}$ are the channel matrices between AP 1 and STA $j$ of AP 2, and between AP 2 and STA $i$ of AP 1 , respectively.

2.3. Eigenvector Transmission. As the transmission weight for the time resource sharing scenario, we employ eigenvector transmission [21] and the water pouring strategies [22]. This is because these techniques maximize the mutual information when the channel state information is available at AP. In eigenvector transmission, the transmission weight at AP $k$ for STA $i$ is calculated as the eigenvectors of the correlation matrix, $\mathbf{H}_{k, i}^{H} \mathbf{H}_{k, i}$. By using singular value decomposition (SVD), the eigenvectors are obtained as the right singular vectors of $\mathbf{H}_{k, i}$ as

$$
\mathbf{H}_{k, i}=\mathbf{U}_{k, i}\left(\begin{array}{ll}
\boldsymbol{\Sigma}_{k, i} & \mathbf{0}
\end{array}\right)\left(\begin{array}{ll}
\mathbf{V}_{k, i}^{(s)} & \mathbf{V}_{k, i}^{(\mathrm{n})}
\end{array}\right)^{H}
$$

where $\mathbf{V}_{k, i}^{(s)} \in \mathbb{C}^{N \times M}$ and $\mathbf{V}_{k, i}^{(n)} \in \mathbb{C}^{N \times(N-M)}$ represent the right singular vectors for the signal space and the null space, respectively. The diagonal elements of $\boldsymbol{\Sigma}_{k, i}$ are the square roots of $\lambda_{k, 1}, \lambda_{k, 2}, \ldots, \lambda_{k, M}$, which are the eigenvalues of $\mathbf{H}_{k, i}^{H} \mathbf{H}_{k, i}$. In eigenvector transmission, transmission weight $\mathbf{W}_{k, i}$ can be chosen as $L_{k, i}$ vectors of $\mathbf{V}_{k, i}^{(s)}$ corresponding to higher eigenvalues. $L_{k, i}$ and the norm of each vectors of $\mathbf{W}_{k, i}$ are determined by water pouring strategies subject to power constraint, that is, $\operatorname{trace}\left(\mathbf{W}_{k, i}^{H} \mathbf{W}_{k, i}\right)$ is one. By using the right singular vectors as the transmission weight, high SNRs corresponding to the eigenvalues can be obtained. We define the transmission weight in eigenvector transmission as $\mathbf{W}_{\mathrm{E}, k, i}$. In (3), $\mathbf{U}_{k, i}$ denotes the left singular vectors and corresponds to the reception weight at STA $i$ in eigenvector transmission.

2.4. ZF Transmission for Interference AP. When there are two APs using the same frequency and the same time slot, the interference from the other AP degrades the transmission performance. Thus, we consider the transmission weight that prevents the interference to the other AP. In AP $k$, the interference channel matrix for STA $j$ of the other AP, AP $l$, is expressed as

$$
\mathbf{H}_{\mathrm{I}, k, j}=\mathbf{U}_{\mathrm{I}, k, j}\left(\begin{array}{ll}
\boldsymbol{\Sigma}_{\mathrm{I}, k, j} & \mathbf{0}
\end{array}\right)\left(\begin{array}{ll}
\mathbf{V}_{\mathrm{I}, k, j}^{(\mathrm{s})} & \mathbf{V}_{\mathrm{I}, k, j}^{(\mathrm{n})}
\end{array}\right)^{H},
$$

where $\mathbf{V}_{\mathrm{I}, k, j}^{(\mathrm{s})} \in \mathbb{C}^{N \times M}$ and $\mathbf{V}_{\mathrm{I}, k, j}^{(\mathrm{n})} \in \mathbb{C}^{N \times(N-M)}$ represent the right singular vectors for the signal space and the null space, respectively. The diagonal elements of $\boldsymbol{\Sigma}_{\mathrm{I}, k, j}$ are the square roots of $\lambda_{k, 1}, \lambda_{k, 2}, \ldots, \lambda_{k, M}$, which are the null space eigenvalues. To obtain the transmission weight that does not interfere with the transmission of AP $l$, the null space channel matrix, $\mathbf{H}_{k, i} \mathbf{V}_{k, j}^{(\mathrm{n})}$ is decomposed by SVD to yield

$$
\mathbf{H}_{k, i} \mathbf{V}_{\mathrm{I}, k, j}^{(\mathrm{n})}=\mathbf{U}_{\mathrm{N}, k, i}\left(\begin{array}{ll}
\mathbf{\Sigma}_{\mathrm{N}, k, j} & \mathbf{0}
\end{array}\right)\left(\begin{array}{ll}
\mathbf{V}_{\mathrm{N}, k, j}^{(\mathrm{s})} & \mathbf{V}_{\mathrm{N}, k, j}^{(\mathrm{n})}
\end{array}\right)^{H},
$$

where $\mathbf{V}_{\mathrm{N}, k, j}^{(s)} \in \mathbb{C}^{(N-M) \times M}$ and $\mathbf{V}_{\mathrm{N}, k, j}^{(n)} \in \mathbb{C}^{N-M \times(N-2 M)}$ represent the null space right singular vectors for the signal space and the null space, respectively. In ZF transmission, the transmission weight $\mathbf{W}_{k, i}$ can be chosen as $L_{k, i}$ vectors of $\mathbf{V}_{\mathrm{I}, k, i}^{(n)} \mathbf{V}_{\mathrm{N}, k, i}^{(s)}$ corresponding to the higher eigenvalues of $\lambda_{\mathrm{N}, k, i}$. $L_{k, i}$ and the norm of each vector of $\mathbf{W}_{k, i}$ is determined by water pouring strategies using $\lambda_{\mathrm{N}, k, i}$. The transmission weight is expressed as $\mathbf{W}_{\mathrm{Z}, k, i}$.

2.5. Channel State Informationat AP And STA. The CSI is obtained at the transmitter and the receiver. We assume that the STA holds the perfect CSI while the CSI at the AP has some estimation error. The channel matrix obtained at AP $k$ and is expressed as

$$
\begin{gathered}
\overline{\mathbf{H}}_{k, i}=\mathbf{H}_{k, i}+\mathbf{E}_{k, i}, \\
\overline{\mathbf{H}}_{\mathrm{I}, k, j}=\mathbf{H}_{\mathrm{I}, k, j}+\mathbf{E}_{\mathrm{I}, k, j},
\end{gathered}
$$

where $\mathbf{E}_{k, i}$ and $\mathbf{E}_{\mathrm{I}, k, j}$ are all i.i.d zero-mean complex Gaussian with variance of $\sigma_{e}^{2}$. Thus, the transmission weight is calculated using the imperfect channel matrix, $\overline{\mathbf{H}}_{k, i}$ and $\overline{\mathbf{H}}_{\mathrm{I}, k, j}$.

2.6. Achievable Bit Rate. When two APs transmit signals in different time slots, that is, time resource sharing systems, the mutual information between AP $k$ and STA $i$ is expressed using the eigenvector transmission weight as

$$
C_{\mathrm{S}, k, i}=\log _{2}\left|\mathbf{I}_{M}+\mathbf{H}_{k, i} \mathbf{W}_{\mathrm{E}, k, i} \mathbf{W}_{\mathrm{E}, k, i}^{H} \mathbf{H}_{k}^{H}\right|,
$$

where $k$ is 1 or 2 and $i=1,2, \ldots, U$. In this scenario, the simultaneous transmission at both APs is avoided. CSMA/CA and RTS/CTS/ACQ in MAC enables this scenario.

We describe the channel capacity in a spatial resource sharing system with two APs as

$$
\begin{aligned}
C_{\mathrm{M}, i, j}= & \log _{2}\left|\mathbf{I}_{M}+\frac{\mathbf{H}_{1, i} \mathbf{W}_{1, i} \mathbf{W}_{1, i}^{H} \mathbf{H}_{1}^{H}}{\mathbf{I}_{M}+\mathbf{H}_{\mathrm{I}, 2, i} \mathbf{W}_{2, j} \mathbf{W}_{2, j}^{H} \mathbf{H}_{\mathrm{I}, 2, i}^{H}}\right| \\
& +\log _{2}\left|\mathbf{I}_{M}+\frac{\mathbf{H}_{2, j} \mathbf{W}_{2, j} \mathbf{W}_{2, j}^{H} \mathbf{H}_{2, j}^{H}}{\mathbf{I}_{M}+\mathbf{H}_{\mathrm{I}, 2, i} \mathbf{W}_{1, i} \mathbf{W}_{1, i}^{H} \mathbf{H}_{\mathrm{I}, 2, j}^{H}}\right|,
\end{aligned}
$$


where $i=1,2, \ldots, U$, and $j=1,2, \ldots, U$. We consider two scenarios for spatial resource sharing. One is primarysecondary AP method based on zero forcing (PSZ) and the other is cooperative AP method based on zero forcing (CZ). In PSZ mode, AP $k$ determines the transmission weight based on the eigenvector transmission using $\mathbf{V}_{k, i}^{(s)}$ while AP $l(l \neq k)$ uses the transmission weight based on the ZF using $\mathbf{V}_{\mathrm{I}, k, i}^{(n)} \mathbf{V}_{\mathrm{N}, k, i}^{(s)}$. Thus, the transmission of AP $l$ suffers interference from the transmission of AP $k$ and the signal power is degraded due to the null space beamforming, although the transmission of AP $k$ is expected to have the high channel capacity, see (7), due to the suppressed interference at AP $l$. The achievable transmission bit rate in the PSZ method is given as

$$
\begin{aligned}
C_{\mathrm{PSZ}, k, i, j}= & \log _{2}\left|\mathbf{I}_{M}+\frac{\mathbf{H}_{k, i} \mathbf{W}_{\mathrm{E}, k, i} \mathbf{W}_{\mathrm{E}, 1, i}^{H} \mathbf{H}_{1}^{H}}{\mathbf{I}_{M}+\mathbf{H}_{\mathrm{I}, 2, i} \mathbf{W}_{\mathrm{Z}, 2, j} \mathbf{W}_{\mathrm{Z}, 2, j}^{H} \mathbf{H}_{\mathrm{I}, 2, i}^{H}}\right| \\
& +\log _{2}\left|\mathbf{I}_{M}+\frac{\mathbf{H}_{l, j} \mathbf{W}_{\mathrm{Z}, 2, j} \mathbf{W}_{\mathrm{Z}, 2, j}^{H} \mathbf{H}_{l, j}^{H}}{\mathbf{I}_{M}+\mathbf{H}_{\mathrm{I}, l, i} \mathbf{W}_{\mathrm{E}, k, i} \mathbf{W}_{\mathrm{E}, k, i}^{H} \mathbf{H}_{\mathrm{I}, l, j}^{H}}\right|,
\end{aligned}
$$

where $\operatorname{AP} k(k=1,2)$ is the primary AP. If there is no channel estimation error at the AP, (9) is rewritten as

$$
\begin{aligned}
C_{\mathrm{PSZ}, k, i, j}= & \log _{2}\left|\mathbf{I}_{M}+\mathbf{H}_{k, i} \mathbf{W}_{\mathrm{E}, k, i} \mathbf{W}_{\mathrm{E}, 1, i}^{H} \mathbf{H}_{1}^{H}\right| \\
& +\log _{2}\left|\mathbf{I}_{M}+\frac{\mathbf{H}_{l, j} \mathbf{W}_{\mathrm{Z}, 2, j} \mathbf{W}_{\mathrm{Z}, 2, j}^{H} \mathbf{H}_{l, j}^{H}}{\mathbf{I}_{M}+\mathbf{H}_{\mathrm{I}, l, i} \mathbf{W}_{\mathrm{E}, k, i} \mathbf{W}_{\mathrm{E}, k, i}^{H} \mathbf{H}_{\mathrm{I}, l, j}^{H}}\right| .
\end{aligned}
$$

In the cooperating mode, both APs uses the transmission weight based on ZF. This case is expected to yield the high capacity among two APs although the signal powers at the destination STAs are degraded due to ZF beamforming. The achievable transmission bit rate in $\mathrm{CZ}$ method is given as

$$
\begin{aligned}
C_{\mathrm{CZ}, i, j}= & \log _{2}\left|\mathbf{I}_{M}+\frac{\mathbf{H}_{k, i} \mathbf{W}_{\mathrm{Z}, k, i} \mathbf{W}_{\mathrm{Z}, k, i}^{H} \mathbf{H}_{k, i}^{H}}{\mathbf{I}_{M}+\mathbf{H}_{\mathrm{I}, k, i} \mathbf{W}_{\mathrm{Z}, l, j} \mathbf{W}_{\mathrm{Z}, l, j}^{H} \mathbf{H}_{\mathrm{I}, k, i}^{H}}\right| \\
& +\log _{2}\left|\mathbf{I}_{M}+\frac{\mathbf{H}_{l, j} \mathbf{W}_{\mathrm{Z}, l, j} \mathbf{W}_{\mathrm{Z}, l, j}^{H} \mathbf{H}_{l, j}^{H}}{\mathbf{I}_{M}+\mathbf{H}_{\mathrm{I}, l, i} \mathbf{W}_{\mathrm{Z}, k, i} \mathbf{W}_{\mathrm{Z}, k, i}^{H} \mathbf{H}_{\mathrm{I}, l, j}^{H}}\right| .
\end{aligned}
$$

If there is no channel estimation error at the AP, (11) is given as

$$
\begin{aligned}
C_{\mathrm{CZ}, i, j}= & \log _{2}\left|\mathbf{I}_{M}+\mathbf{H}_{k, i} \mathbf{W}_{\mathrm{Z}, k, i} \mathbf{W}_{\mathrm{Z}, k, i}^{H} \mathbf{H}_{k, i}^{H}\right| \\
& +\log _{2}\left|\mathbf{I}_{M}+\mathbf{H}_{l, j} \mathbf{W}_{\mathrm{Z}, l, j} \mathbf{W}_{\mathrm{Z}, \mathrm{l}, j}^{H} \mathbf{H}_{l, j}^{H}\right| .
\end{aligned}
$$

2.7. Intercell Interference. In spatial resource sharing, the ICI determines the channel capacity in (9) and (11). Therefore, the ICI is analyzed by numerical approach. We define the variance of elements of the interference channel matrix, $\mathbf{H}_{\mathrm{I}, k, i}$, as $\gamma_{\mathrm{I}}$ to show the characteristics of the ICI. When

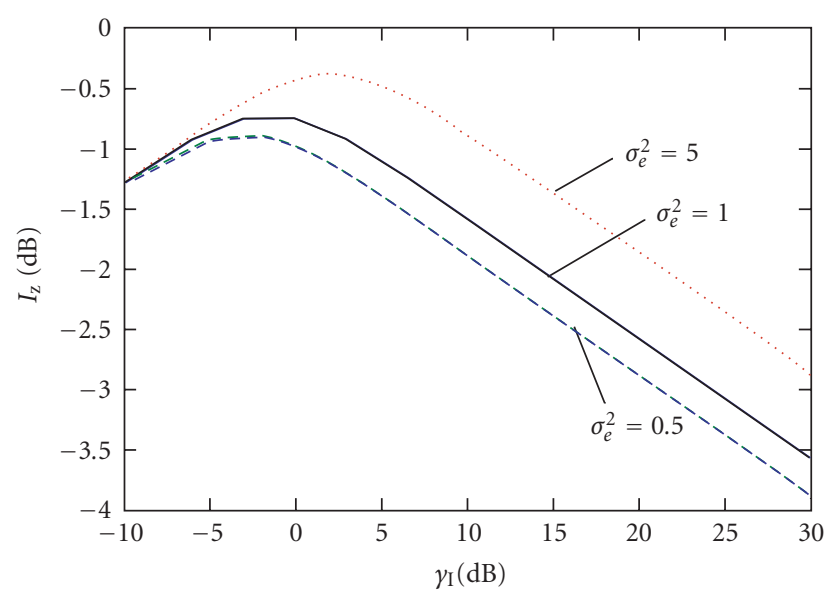

FIGURE 1: Intercell interference power versus $\gamma_{\mathrm{I}}$.

the eigenvector transmission is applied, the ICI power, $I_{E}$, is expressed as

$$
\begin{aligned}
I_{\mathrm{E}} & =\operatorname{trace}\left(\mathbf{W}_{\mathrm{E}, k, i}^{H} \mathbf{H}_{\mathrm{I}, l, j}^{H} \mathbf{H}_{\mathrm{I}, l, j} \mathbf{W}_{\mathrm{E}, k, i}\right) \\
& =\operatorname{trace}\left(\mathbf{W}_{\mathrm{E}, k, i}^{H} \mathbf{V}_{\mathrm{Z}, l, j}^{(s)} \Sigma_{\mathrm{I}, l, j}^{(s)} \mathbf{U}_{\mathrm{I}, l, j}^{H} \mathbf{U}_{\mathrm{I}, l, j} \Sigma_{\mathrm{I}, l, j}^{(s)} \mathbf{V}_{\mathrm{Z}, l, j}^{(s) H} \mathbf{W}_{\mathrm{E}, k, i}\right) \\
& =\operatorname{trace}\left(\mathbf{W}_{\mathrm{E}, k, i}^{H} \mathbf{V}_{\mathrm{Z}, l, j}^{(s)} \boldsymbol{\Sigma}_{\mathrm{I}, l, j}^{(s) 2} \mathbf{V}_{\mathrm{Z}, l, j}^{(s) H} \mathbf{W}_{\mathrm{E}, k, i}\right) \\
& =\operatorname{trace}\left(\mathbf{A}^{H} \boldsymbol{\Sigma}_{\mathrm{I}, l, j}^{(s) 2} \mathbf{A}\right),
\end{aligned}
$$

where $\Sigma_{\mathrm{I}, l, j}^{(s)} \in \mathbb{C}^{M \times M}$ is the diagonal matrix for the signal space and $\mathbf{A}=\mathbf{V}_{\mathrm{Z}, l, j}^{(s) H} \mathbf{W}_{\mathrm{E}, k, i}$. Since the $\mathbf{V}_{\mathrm{Z}, l, j}^{(s)}$ is not correlate to $\mathrm{W}_{\mathrm{E}, k, i}, I_{\mathrm{E}}$ is determined by $\gamma_{\mathrm{I}}$ regardless of the channel estimation error.

Then, the ICI power for ZF weight, $I_{Z}$, is expressed as

$$
\begin{aligned}
I_{\mathrm{Z}} & =\operatorname{trace}\left(\mathbf{W}_{\mathrm{Z}, l, j}^{H} \mathbf{H}_{\mathrm{I}, l, j}^{H} \mathbf{H}_{\mathrm{I}, l, i} \mathbf{W}_{\mathrm{Z}, l, j}\right) \\
& =\operatorname{trace}\left(\overline{\mathbf{V}}_{\mathrm{N}, l, i}^{(s) H} \overline{\mathbf{V}}_{\mathrm{Z}, l, i}^{(n) H} \mathbf{V}_{\mathrm{Z}, l, i}^{(s)} \boldsymbol{\Sigma}_{\mathrm{I}, l, i}^{(s) 2} \mathbf{V}_{\mathrm{Z}, l, i}^{(s) H} \overline{\mathbf{V}}_{\mathrm{Z}, l, i}^{(n)} \overline{\mathbf{V}}_{\mathrm{N}, l, i}^{(s)}\right) \\
& =\operatorname{trace}\left(\mathbf{B}^{H} \boldsymbol{\Sigma}_{\mathrm{I}, l, i}^{(s) 2} \mathbf{B}\right)
\end{aligned}
$$

where $\overline{\mathbf{V}}_{\mathrm{Z}, k, j}^{(n)} \in \mathbb{C}^{N \times(N-M)}$ and $\overline{\mathbf{V}}_{\mathrm{N}, k, j}^{(s)} \in \mathbb{C}^{(N-M) \times L_{K, j}}$ are the right singular vectors for null space and null space right singular vectors for signal space calculated by using the channel matrices with channel estimation error. $\mathbf{B}=$ $\mathbf{V}_{\mathrm{Z}, l, i}^{(s) H} \overline{\mathbf{V}}_{\mathrm{Z}, l, i}^{(n) H} \overline{\mathbf{V}}_{\mathrm{N}, l, i}^{(s)}$. In the case without the channel estimation error, $I_{Z}=0$ since $\mathbf{V}_{Z, k, j}^{(s) H} \overline{\mathbf{V}}_{Z, k, j}^{(n)}=\mathbf{0}$. Since the norm of $\mathbf{B}$ increases as the channel estimation error becomes large, $I_{Z}$ is affected by $\gamma_{\mathrm{I}}$ and channel estimation error, $\sigma_{e}^{2}$. The ratio of channel estimation error, $\sigma_{e}^{2}$, to $\gamma_{\mathrm{I}}$ increases as $\gamma_{\mathrm{I}}$ decreases. Therefore, the norm of $\mathbf{B}$ and $\gamma_{\mathrm{I}}$ have inverse correlation. Figure 1 shows $I_{Z}$ versus $\gamma_{\mathrm{I}}$ when the estimation error, $\sigma_{e}^{2}$, is set to be $0.5,1.0$, and 5.0 times the noise variance, $\sigma^{2}$, of one. We can see that the local maximal value and $\gamma_{\mathrm{I}}$ of the local maximal become large the channel estimation error increases. When $\gamma_{\mathrm{I}} \gg 0 \mathrm{~dB}$, 

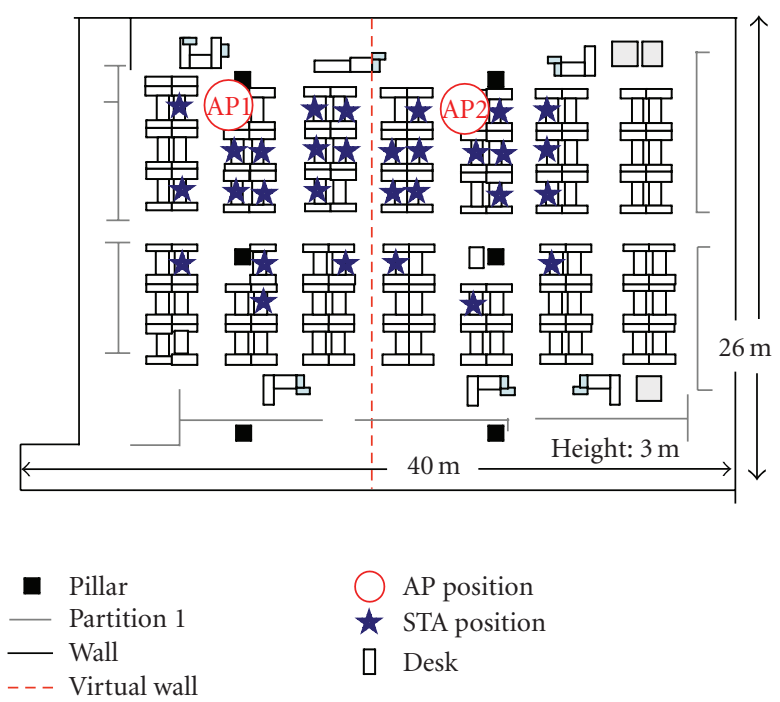

Figure 2: Experimental environment.
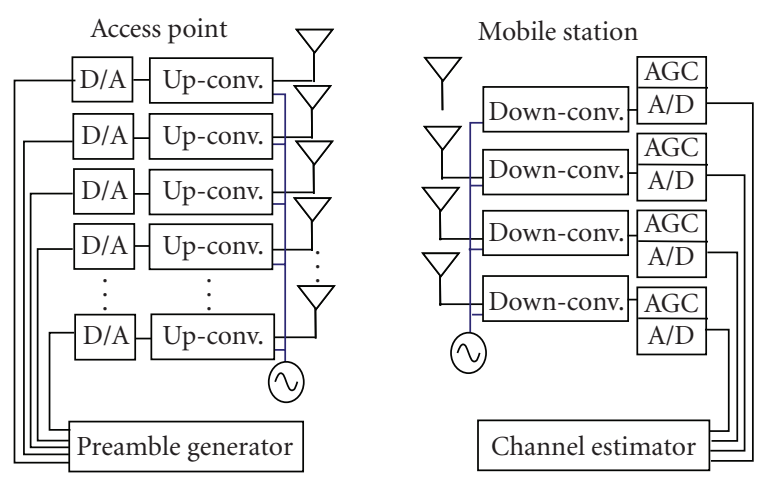

FIGURE 3: Block diagram of the MIMO testbed.

$\mathrm{ZF}$ works well since $I_{\mathrm{Z}}$ mitigation is proportional to the interference channel norm, $\gamma_{\mathrm{I}}$. However, when $\gamma_{\mathrm{I}}$ is less than $5 \mathrm{~dB}$, the transmission performance of spatial resource sharing based on ZF may be degraded. To estimate the realistic transmission performance, we measured the channel matrices, $\mathbf{H}$, and the interference channel matrices, $\mathbf{H}_{\mathrm{I}}$, in an actual indoor environment and evaluated the effectiveness of space resource sharing by using the measured channel.

\section{Channel Condition}

3.1. Measured Channel Matrix. We measured the channel matrices in an actual indoor environment using the testbed for MIMO-OFDM transmission. The measurement environment is the $40 \times 26 \times 3[\mathrm{~m}]$ room shown in Figure 2 . We used the channel matrices measured in an actual environment to evaluate the transmission performance of spatial resource sharing systems.

In the experiment, we used a circular array of eight antennas with element spacing of $1.0 \lambda$ and a four-antenna linear array with element spacing of $0.5 \lambda$ as the transmit antennas and the receive antennas, respectively. Therefore,
TABLE 1: Parameters of MIMO-OFDM testbed.

\begin{tabular}{lc}
\hline Number of antennas & $8(\mathrm{Tx}), 4(\mathrm{Rx})$ \\
Radio frequency & $4.85 \mathrm{GHz}$ \\
Bandwidth & $20 \mathrm{MHz}$ \\
Total transmission power & $10 \mathrm{dBm}$ \\
Sensitivity & -20 to $-70 \mathrm{dBm}$ \\
Sampling rate & $20 \mathrm{MHz}(\mathrm{A} / \mathrm{D}), 80 \mathrm{MHz}(\mathrm{D} / \mathrm{A})$ \\
Number of FFT points & 64 \\
Number of subcarriers & $48($ Information $)+4(\mathrm{Pilot})$ \\
Short preamble length & $0.8 \mu \mathrm{sec} \times 10=8 \mu \mathrm{sec}$ \\
OFDM symbol length & $3.2 \times 2 \mu \mathrm{s}+1.6 \mu \mathrm{s}(\mathrm{GI})$ \\
\hline
\end{tabular}

we obtained $8 \times 4$ MIMO channel matrices. We consider $8 \times 4$ and $8 \times 2$ MIMO systems and $8 \times 2$ MIMO channel matrices are obtained using the center two receive antennas of the linear array. The AP was established on a $2.5 \mathrm{~m}$ pole and located at points AP 1, and AP 2 in Figure 2. The receive antenna array was attached to a laptop computer on a desk $0.7 \mathrm{~m}$ high that was placed at the star marks in Figure 2. The partitions are made of metal and partition height is $1.9 \mathrm{~m}$. The each desk is separated by $1.4 \mathrm{~m}$ metal barriers.

A block diagram of the experimental testbed is shown in Figure 3 and the main parameters for this testbed are given in Table 1 . The center radio frequency is $4.85 \mathrm{GHz}$ and the OFDM signal is transmitted in $20 \mathrm{MHz}$ bandwidth. The AP transmits the preamble based on the short and long preambles in the IEEE 802.11a standard [10]. The STA estimates the channel matrices of the 48 sub-carriers from the received signals at 30 STA positions. We assume that the left 15 STAs and right 15 STAs communicate with AP 1 and AP 2 , respectively. Thus, the measured channel matrices at AP 1 corresponding to the right 15 STAs and the left 15 STAs are $\mathbf{H}_{1,1}, \mathbf{H}_{1,2}, \ldots, \mathbf{H}_{1,15}$, and $\mathbf{H}_{\mathrm{I}, 1,1}, \mathbf{H}_{\mathrm{I}, 1,2}, \ldots, \mathbf{H}_{\mathrm{I}, 1,15}$, respectively. In AP $2, \mathbf{H}_{2,1}, \mathbf{H}_{2,2}, \ldots, \mathbf{H}_{2,15}$ and $\mathbf{H}_{\mathrm{I}, 2,1}, \mathbf{H}_{\mathrm{I}, 2,2}, \ldots, \mathbf{H}_{\mathrm{I}, 2,15}$, are obtained between AP 2 and the right STAs and left STAs, respectively.

In this experiment, a virtual wall is considered between the AP 1's STAs and AP 2's STAs. Thus, we define the interference channel matrices as $\alpha \mathbf{H}_{\mathrm{I}, 2,1}, \alpha[\mathrm{dB}]$ is the attenuation level of the virtual wall. When $\alpha=0 \mathrm{~dB}$, we use measured channel matrix, $\mathbf{H}_{\mathrm{I}, k, i}$ as the interference channel matrix. By using $\alpha$, we can evaluate the performance of spatial resource sharing corresponding to the distance between two APs or the attenuation level of the wall. The averaged SNRs at STA corresponding to the two APs are shown in Figures 4(a) and 4(b). We assume that the measured channel matrices are accurate regardless of their SNR since the measured channel matrices are obtained by averaging a sufficient number of channel matrices.

\section{Numerical Results}

4.1. Achievable Transmission Bit Rate for i.i.d Channel. First, we consider that $\mathbf{H}_{k, i}$ is a complex Gaussian variable with zero mean (random i.i.d.). The variance of elements of $\mathbf{H}_{k, i}$, $\gamma_{\mathrm{S}}$, is set to be 30 or $10 \mathrm{~dB}$, and the variance of elements of 


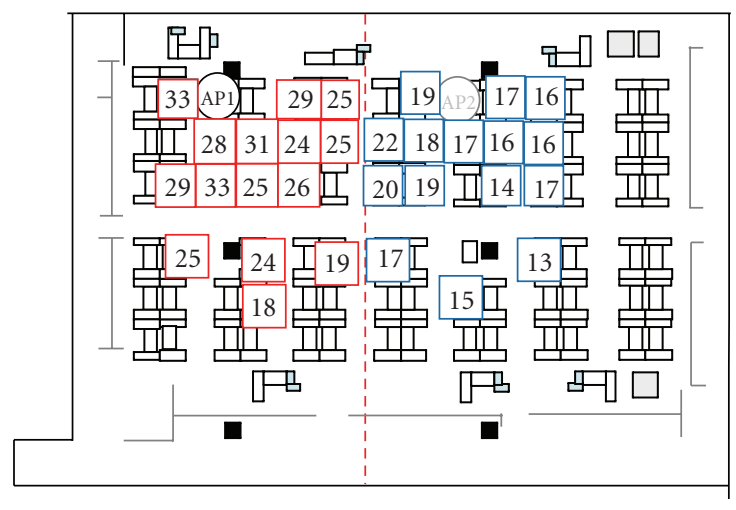

(a)

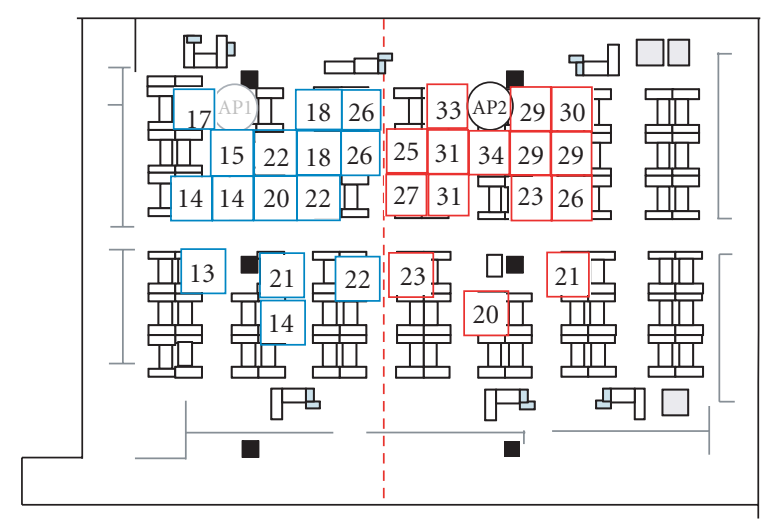

(b)

FIGURE 4: SNR distribution in an experimental environment corresponding to (a) AP 1 and (b) AP 2.

$\mathbf{H}_{\mathrm{I}, k, i}, \gamma_{\mathrm{I}}$, is determined from -5 to $30 \mathrm{~dB}$. Since we assumed a noise variance of one, $\gamma_{\mathrm{S}}$ and $\gamma_{\mathrm{I}}$ express the expectation of the received signal to noise ratio (SNR) and interference to noise ratio (INR). Hereinafter, we take $\gamma_{\mathrm{s}}$ and $\gamma_{\mathrm{I}}$ as the SNR and INR, respectively. The achievable transmission bit rate corresponding to $\gamma_{\mathrm{I}}$ is calculated as the median value of 1,000 trials.

Figure 5(a) plots the median value of the achievable transmission bit rate for $8 \times 4$ MIMO systems versus the INR when the SNR is set to be $30 \mathrm{~dB}$. The achievable transmission bit rate for the eigenvector transmission in the time resource sharing scenario and two spatial resource sharing scenarios, PSZ and CZ methods, are shown as $C_{S}$, $C_{\mathrm{PSZ}}$, and $C_{\mathrm{CZ}}$, respectively. The solid, dashed, and dotted lines represent the achievable transmission bit rate without channel estimation error and with channel estimation error, $\sigma_{e}^{2}=1$ and 5, respectively. It is found that the spatial resource sharing systems have much higher achievable transmission bit rate than that in time resource sharing systems. When the INR is $30 \mathrm{~dB}, C_{C Z}$ is 1.72 times greater than $C_{S}$. When the INR is less than $2.8 \mathrm{~dB}, C_{\mathrm{PSZ}}$ without channel estimation error outperforms $C_{\mathrm{CZ}}$ without channel estimation error. When there are channel estimation error, the achievable transmission bit rates in spatial resource sharing degrade while the degradation by the channel estimation error in $C_{S}$ is negligible. We can see that the intersection of $C_{\mathrm{PSZ}}$ and $C_{\mathrm{CZ}}$ becomes larger as channel estimation error increases. The range where $C_{\mathrm{PSZ}}$ outperforms $C_{\mathrm{CZ}}$ becomes large with large channel estimation error. When there is no channel estimation error, the achievable transmission bit rate of the primary AP in PSZ equals that in $C_{S}$. Therefore, the difference between $C_{\mathrm{PSZ}}$ and $C_{\mathrm{S}}$ denotes the achievable transmission bit rate of the secondary AP in PSZ. Since the interference from the primary AP decreases as the INR becomes small, the achievable transmission bit rate in PSZ increases.

Figure 5(b) plots the median value of the achievable transmission bit rate when SNR is $10 \mathrm{~dB}$. In Figure 5(b), $C_{\mathrm{CZ}}$ is 1.42 times greater than $C_{S}$ for the INR are $30 \mathrm{~dB}$. The intersection of $C_{\mathrm{PSZ}}$ and $C_{\mathrm{CZ}}$ with channel estimation error decreases compared to that in Figure 5(a). We can see that the local minimal value of $C_{\mathrm{CZ}}$ with channel estimation error in Figures 5(a) and 5(b) corresponds to the local maximal of $I_{z}$ in Figure 1 and that the degradation is large when the SNR is small.

Figures 6(a) and 6(b) plot that the median values of the achievable transmission bit rate for $8 \times 2$ MIMO systems for SNR values of $30 \mathrm{~dB}$ and $10 \mathrm{~dB}$, respectively. Figures 6(a) and 6(b) shows that $C_{\mathrm{CZ}}$ is 1.91 times and 1.80 times greater than $C_{S}$ when the INR is $30 \mathrm{~dB}$ and $10 \mathrm{~dB}$, respectively. Spatial resource sharing is more effective in $8 \times 2$ MIMO systems than in $8 \times 4$ MIMO systems. This is because the fewer receive antennas of the STA corresponding to the interference channel matrix increases the dimension of $\mathbf{V}_{\mathrm{I}, k, j}^{(n)} \in \mathbb{C}^{N \times(N-M)}$ in (4). $\mathbf{V}_{\mathrm{I}, k, j}^{(n)}$ with large dimension values prevents the null space eigenvalues in (5) from decreasing compared to the eigenvalues in (3).

4.2. Achievable Transmission Bit Rate for Indoor Environment. The achievable transmission bit rate is calculated using 2 (AP positions) $\times 48$ (subcarriers) $\times 10$ (MS positions) measured channel matrices. Figures 7 and 8 show the cumulative probability of the achievable transmission bit rate in $8 \times 4$ and $8 \times 2$ MIMO systems. The solid, dashed, and dotted lines denote the achievable transmission bit rate with channel estimation error, $\sigma_{e}^{2}=0, \sigma_{e}^{2}=1$, and $\sigma_{e}^{2}=5$, respectively. In this section, we does not use the virtual wall attenuation, $\alpha=0[\mathrm{~dB}]$. In $8 \times 4$ MIMO systems without the channel estimation error, the median values of $C_{\mathrm{CZ}}$ and $C_{\mathrm{SPZ}}$ are 1.59 and 1.38 times greater than that of $C_{S}$, respectively. The median values of $C_{\mathrm{CZ}}$ and $C_{\mathrm{SPZ}}$ in $8 \times 2 \mathrm{MIMO}$ systems without channel estimation error are 1.90 and 1.51 times greater than that of $C_{S}$, respectively. The degradation in the achievable transmission bit rate due to the channel estimation error in $8 \times 4$ MIMO systems is larger than that in $8 \times 2 \mathrm{MIMO}$ systems. Figures 7 and 8 show that the median values of $C_{\mathrm{CZ}}$ and $C_{\mathrm{PSZ}}$ in $8 \times 4(8 \times 2) \mathrm{MIMO}$ systems with $\sigma_{e}^{2}$ of one and five are 1.41 (1.74) and 1.19 (1.51) times greater than that of $C_{S}$. The decrease in $C_{\mathrm{CZ}}$ due to the channel 


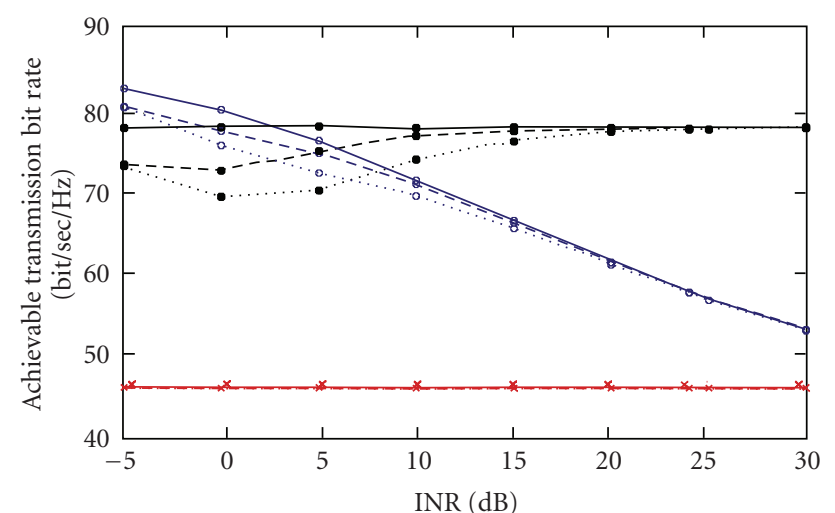

(a)

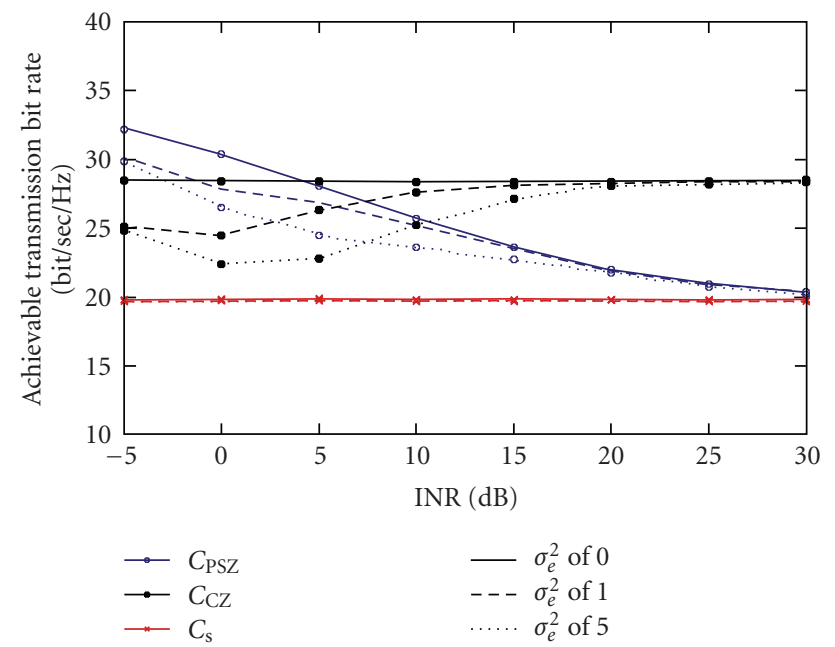

(b)

Figure 5: Median value of achievable transmission bit rate versus INR for $8 \times 4$ MIMO systems (a) when SNR is $30 \mathrm{~dB}$ and (b) when SNR is $10 \mathrm{~dB}$.

estimation error is large compared to the results in Figures 5 and 6 . It is found that the spatial resource sharing with large channel estimation error, $\sigma_{e}^{2}$ of one or five, high achievable transmission bit rates are obtained compared to $C_{\mathrm{S}}$.

4.3. Virtual Wall Attenuation. Figures 9 and 10 show the median value of the achievable transmission bit rate versus the virtual wall attenuation factor, $\alpha$, for $8 \times 4$ and $8 \times 2 \mathrm{MIMO}$ systems, respectively. The tendencies of Figures 9 and 10 are similar to $C_{\mathrm{PSZ}}$ and $C_{\mathrm{CZ}}$ of Figures 5 and 6 . $C_{\mathrm{PSZ}}$ becomes large as the virtual wall attenuation factor decreases. In $8 \times$ 4 MIMO systems without channel estimation error, $C_{\mathrm{PSZ}}$ outperforms $C_{\mathrm{CZ}}$ when $\alpha$ is less than $-10 \mathrm{~dB}$. As the channel estimation error increases, the intersection of $C_{\mathrm{PSZ}}$ and $C_{\mathrm{CZ}}$ increases. When there are large channel estimation error, PSZ method becomes effective compared to $\mathrm{CZ}$ method.

In $8 \times 2$ MIMO systems, CZ method is effective. When the estimation error, $\sigma_{e}^{2}$, is one, $C_{\mathrm{CZ}}$ is greater than $C_{\mathrm{PSZ}}$ when $\alpha$ is larger than $-15 \mathrm{~dB}$. Although the spatial resource sharing is affected by channel estimation error, it is found that CZ and PSZ method outperforms time resource sharing

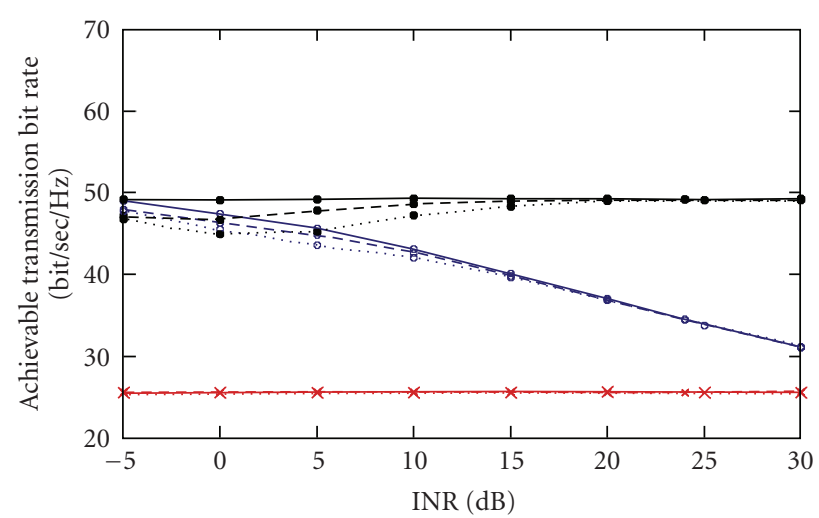

(a)

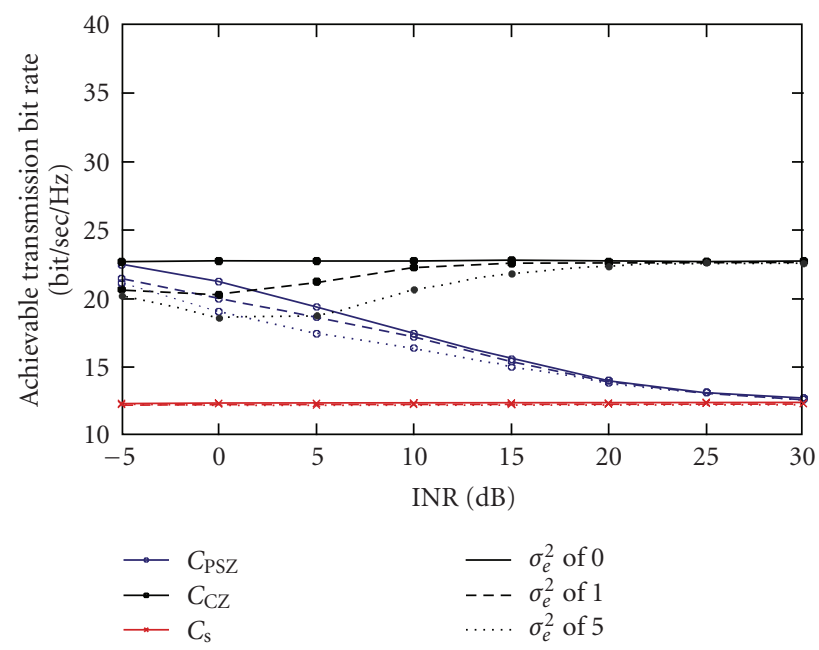

(b)

FIGURE 6: Median value of achievable transmission bit rate versus INR for $8 \times 2$ MIMO systems (a) when SNR is $30 \mathrm{~dB}$ and (b) when SNR is $10 \mathrm{~dB}$.

method even though there are channel estimation error whose variance, $\sigma_{e}^{2}$, is less than five.

Although Figures 5 and 6 show the local minimum points of $C_{\mathrm{CZ}}$ corresponding to the local maximum interference power (see Figure 1), $C_{C Z}$ in Figures 9 and 10 has the local minimum points around $\alpha=0$. This is because there are gaps between the expectations of the channel matrix norm and interference channel matrix norm as shown in Figures 4(a) and 4(b), and the eigenvalue distribution in an actual environment channel is different from that in random i.i.d. channel. It is found that the primary secondary AP is robust against CSI error compared with the cooperative $\mathrm{AP}$ scenario, and $\mathrm{C}_{\mathrm{PSZ}}$ and $\mathrm{C}_{\mathrm{CZ}}$ outperform $\mathrm{C}_{\mathrm{CZ}}$ even when $\alpha=0$.

\section{Conclusion}

We evaluated the transmission performance in spatial resource sharing. This paper introduced the primarysecondary AP scenario based on zero forcing (PSZ) and 


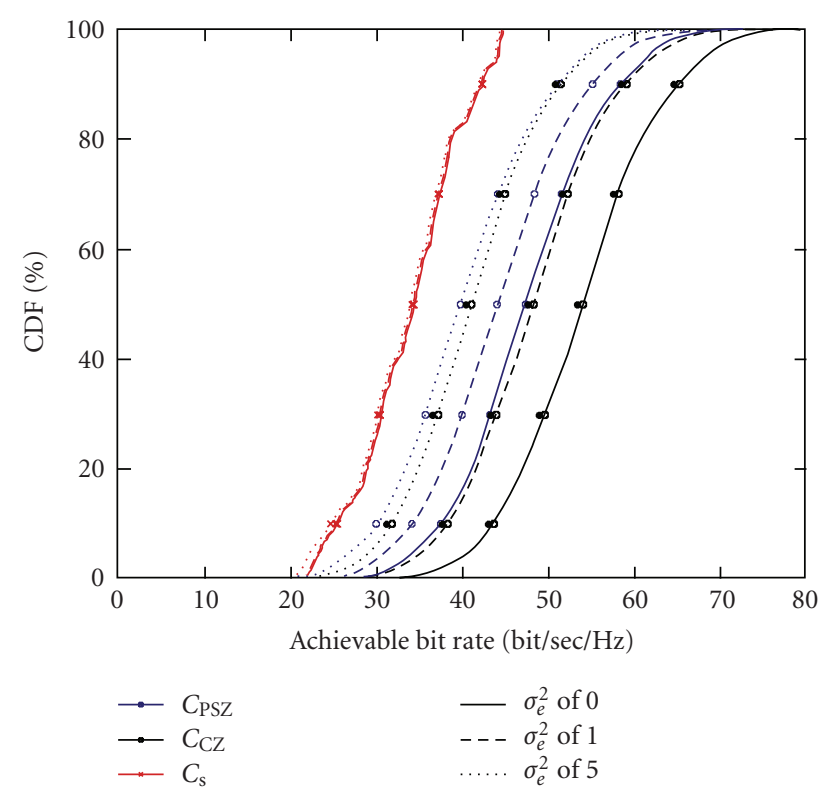

FIGURE 7: CDF of achievable bit rate using measured $8 \times 4$ channel matrix with $\alpha=0$.

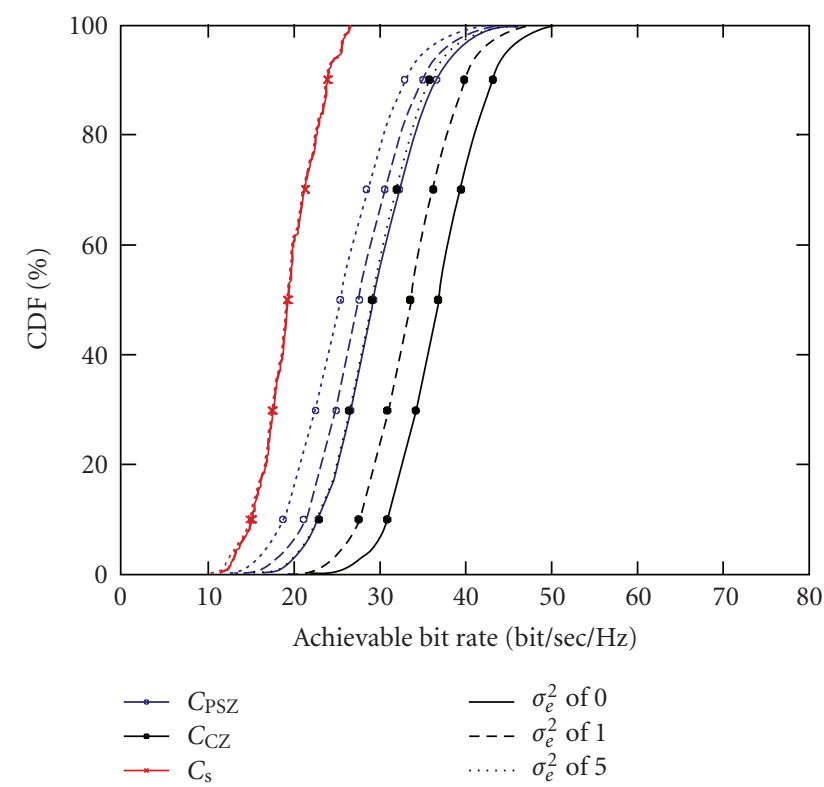

FIGURE 8: CDF of achievable bit rate using measured $8 \times 2$ channel matrix with $\alpha=0$.

cooperative AP scenario based on zero forcing (CZ). Simulation results clarify the difference of applicability domain of these scenarios and the achievable transmission bit rate improvement compared to time domain resource sharing scenario. By using the channel matrices measured in an actual indoor environment, CZ and PSZ methods showed that they yielded 1.59 (1.90) times and 1.38 (1.51) times the achievable transmission bit rate of the time domain resource sharing system for $8 \times 4$ MIMO $(8 \times 2 \mathrm{MIMO})$ systems when there is no channel estimation error. Even though we considered large estimation error, $\sigma_{e}^{2}$ of one, the median

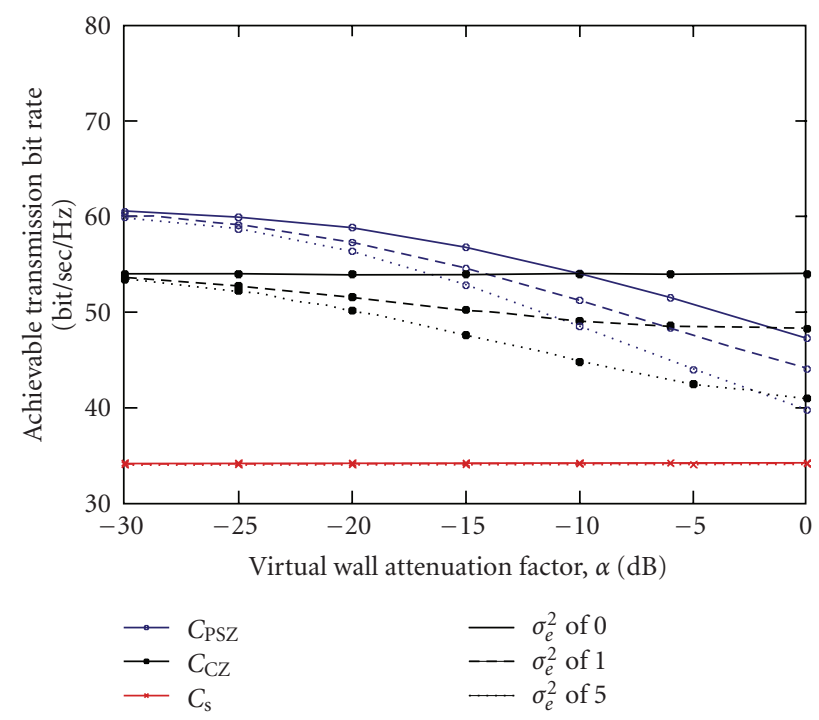

FIGURE 9: Median value of achievable bit rate using measured $8 \times 4$ channel matrices.

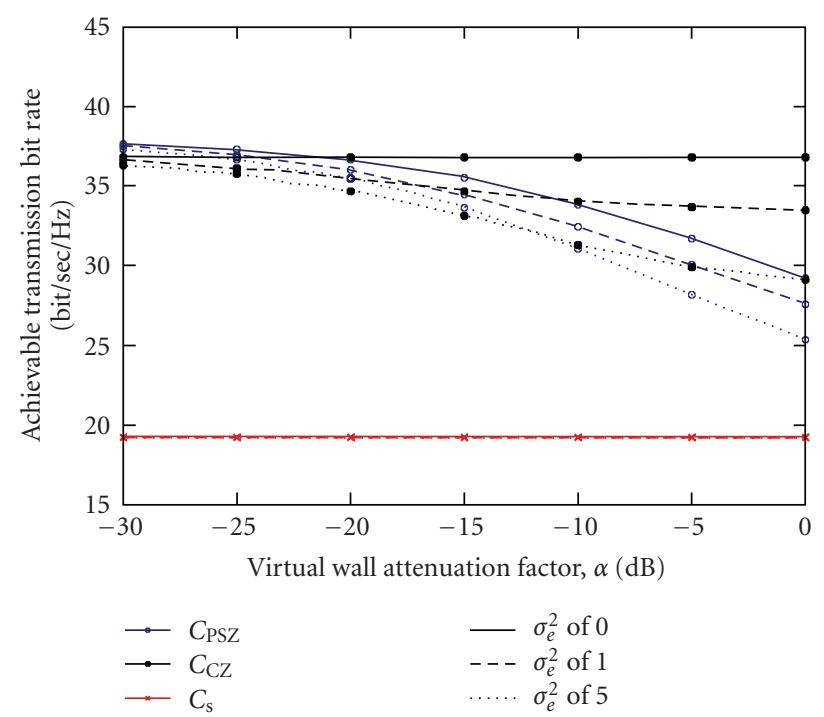

FIGURE 10: Median value of achievable bit rate using measured $8 \times 2$ channel matrix.

values of $C_{\mathrm{CZ}}$ and $C_{\mathrm{PSZ}}$ in $8 \times 4 \mathrm{MIMO}(8 \times 2 \mathrm{MIMO})$ systems with $\sigma_{e}^{2}$ of one and five were $1.41(1.74)$ and 1.19 (1.51) times greater than that of $C_{S}$. By using a virtual wall attenuation factor, it is found that $\mathrm{CZ}$ method is effective in the case where mobile stations (STAs) have small number of the receive antennas and the norm of interference channel matrix is large while PSZ method outperforms CZ method when the norm of interference channel matrix becomes small and CSI error increases. We confirmed that spatial resource sharing is very attractive for the overlapping cell scenario. 


\section{Acknowledgments}

The authors thank Mr. Kazuyasu Okada, the Executive Manager of NTT Network Innovation Laboratories, for his constant encouragement. The authors would also like to thank Mr. Yoshinobu Makise and Mr. Masaaki Ida of NTT Advanced Technology Corporation for their support.

\section{References}

[1] E. Perahia and R. Stacey, Next Generation Wireless LANs: Throughput, Robustness, and Reliability in 802.11n, Cambridge University Press, Cambridge, UK, 2008.

[2] L. M. Correia, Ed., Mobile Broadband Multimedia Networks Techniques, Models and Tools for 4G, Elsevier, New York, NY, USA, 2006.

[3] Information and Communications in Japan, White Paper, Ministry of Internal Affairs and Communications, Tokyo, Japan, 2008.

[4] A. Adler and M. Salhov, "A carrier-crade wireless LAN network implementation," IEEE Microwave Magazine, vol. 9, no. 4, pp. 108-119, 2008.

[5] G. Breit, et al., "TGac Channel Model Addendum," Doc. IEEE802.11-09/0308r5, May 2009.

[6] Y. Takatori, "Importance of Overlapped BSS issue in 802.11ac," Doc. IEEE802.11-09/0630r1, May 2009.

[7] E. Ziouva and T. Antonakopoulos, "CSMA/CA performance under high traffic conditions: throughput and delay analysis," Computer Communications, vol. 25, no. 3, pp. 313-321, 2002.

[8] P. Karn, "MACA: a new channel access method for packet radio," in Proceedings of the Computer Networking Conference, pp. 134-140, September 1990.

[9] I. Katzela and M. Naghshineh, "Channel assignment schemes for cellular mobile telecommunication systems: a comprehensive survey," IEEE Personal Communications, vol. 3, no. 3, pp. 10-31, 1996.

[10] H. Ekström, A. Furuskär, J. Karlsson, et al., "Technical solutions for the $3 \mathrm{G}$ long-term evolution," IEEE Communications Magazine, vol. 44, no. 3, pp. 38-45, 2006.

[11] E. Perahia, "IEEE 802.11 n development:history, process, and technology," IEEE Communications Magazine, vol. 46, no. 7, pp. 48-55, 2008.

[12] Q. H. Spencer, C. B. Peel, A. L. Swindlehurst, and M. Haardt, "An introduction to the multi-user MIMO downlink," IEEE Communications Magazine, vol. 42, no. 10, pp. 60-67, 2004.

[13] K. Nishimori, R. Kudo, N. Honma, Y. Takatori, O. Atsushi, and K. Okada, "Experimental evaluation using $16 \times 16$ multiuser MIMO testbed in an actual indoor scenario," in Proceedings of IEEE International Symposium on Antennas and Propagation (APS '08), pp. 1-4, July 2008.

[14] Y. J. Zhang and K. B. Letaief, "An efficient resource-allocation scheme for spatial multiuser access in MIMO/OFDM systems," IEEE Transactions on Communications, vol. 53, no. 1, pp. 107-116, 2005.

[15] K. B. Letaief and Y. J. Zhang, "Dynamic multiuser resource allocation and adaptation for wireless systems," IEEE Wireless Communications, vol. 13, no. 4, pp. 38-47, 2006.

[16] S. Bellofiore, C. A. Balanis, J. Foutz, and A. S. Spanias, "Smartantenna systems for mobile communication networks-part 1: overview and antenna design," IEEE Antennas and Propagation Magazine, vol. 44, no. 3, pp. 145-154, 2002.
[17] H. Dahrouj and W. Yu, "Coordinated beamforming for the multi-cell multi-antenna wireless system," in in Proceedings of the 42nd Annual Conference on Information Sciences and Systems (CISS '08), pp. 429-434, Princeton, NJ, USA, March $19-21,2008$.

[18] V. Chandrasekhar, J. G. Andrews, and A. Gatherer, "Femtocell networks: a survey," IEEE Communications Magazine, vol. 46, no. 9, pp. 59-67, 2008.

[19] Y. Takatori, F. Fitzek, K. Tsunekawa, and R. Prasad, "Channel capacity of TDD-OFDM-MIMO for multiple access points in a wireless single-frequency-network," Wireless Personal Communications, vol. 35, no. 1-2, pp. 19-33, 2005.

[20] R. Kudo, Y. Takatori, K. Nishimori, A. Ohta, and S. Kubota, "New downlink beamforming method for cooperative multiple access point systems," IEICE Transactions on Communications, vol. E90-B, no. 9, pp. 2303-2311, 2007.

[21] A. Paulraj, R. Nabar, and D. Gore, Introduction to SpaceTime Wireless Communications, Cambridge University Press, Cambridge, UK, 2003.

[22] E. Telatar, "Capacity of multi-antenna gaussian channels," European Transactions on Telecommunications, vol. 10, no. 6, pp. 585-595, 1999. 

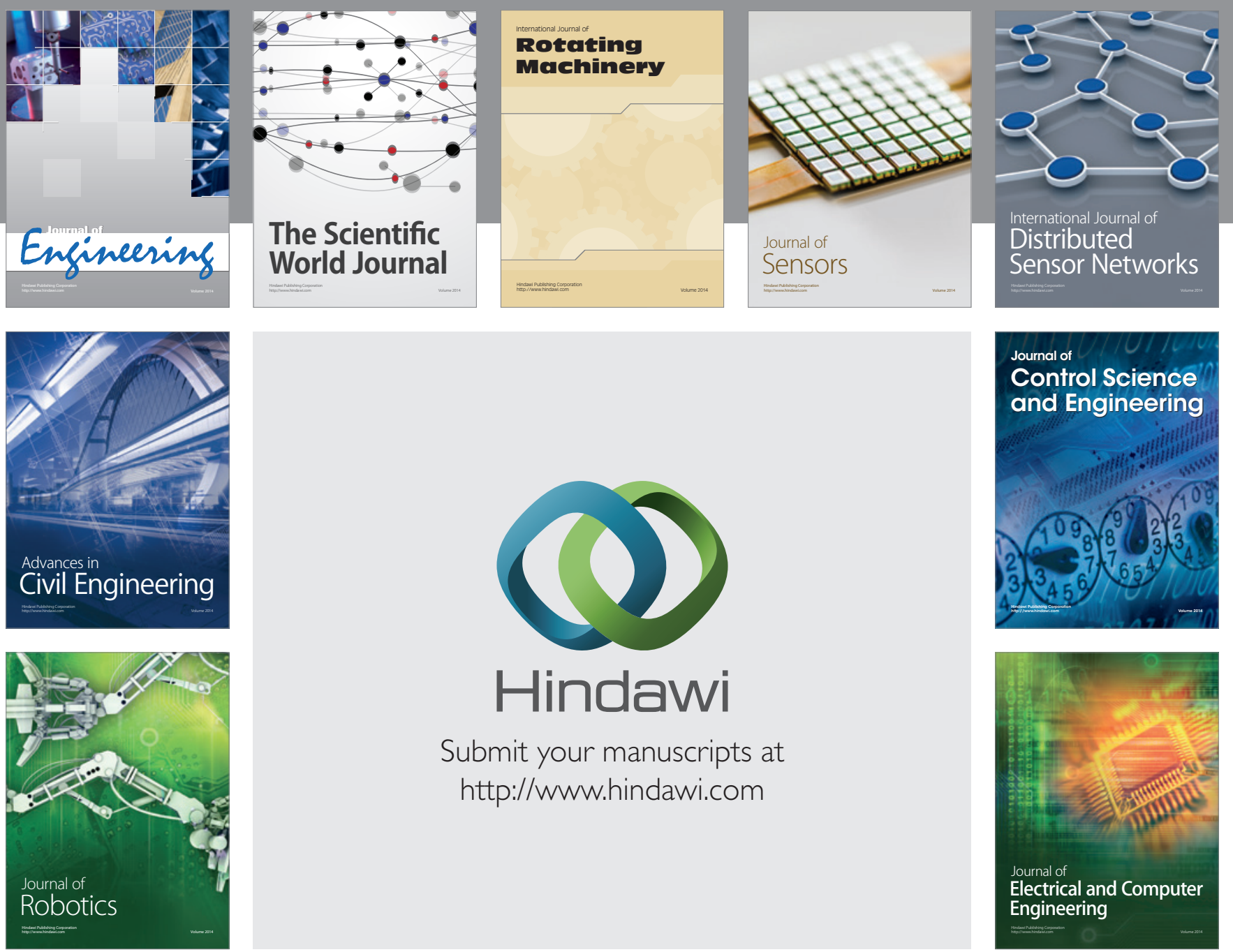

Submit your manuscripts at

http://www.hindawi.com
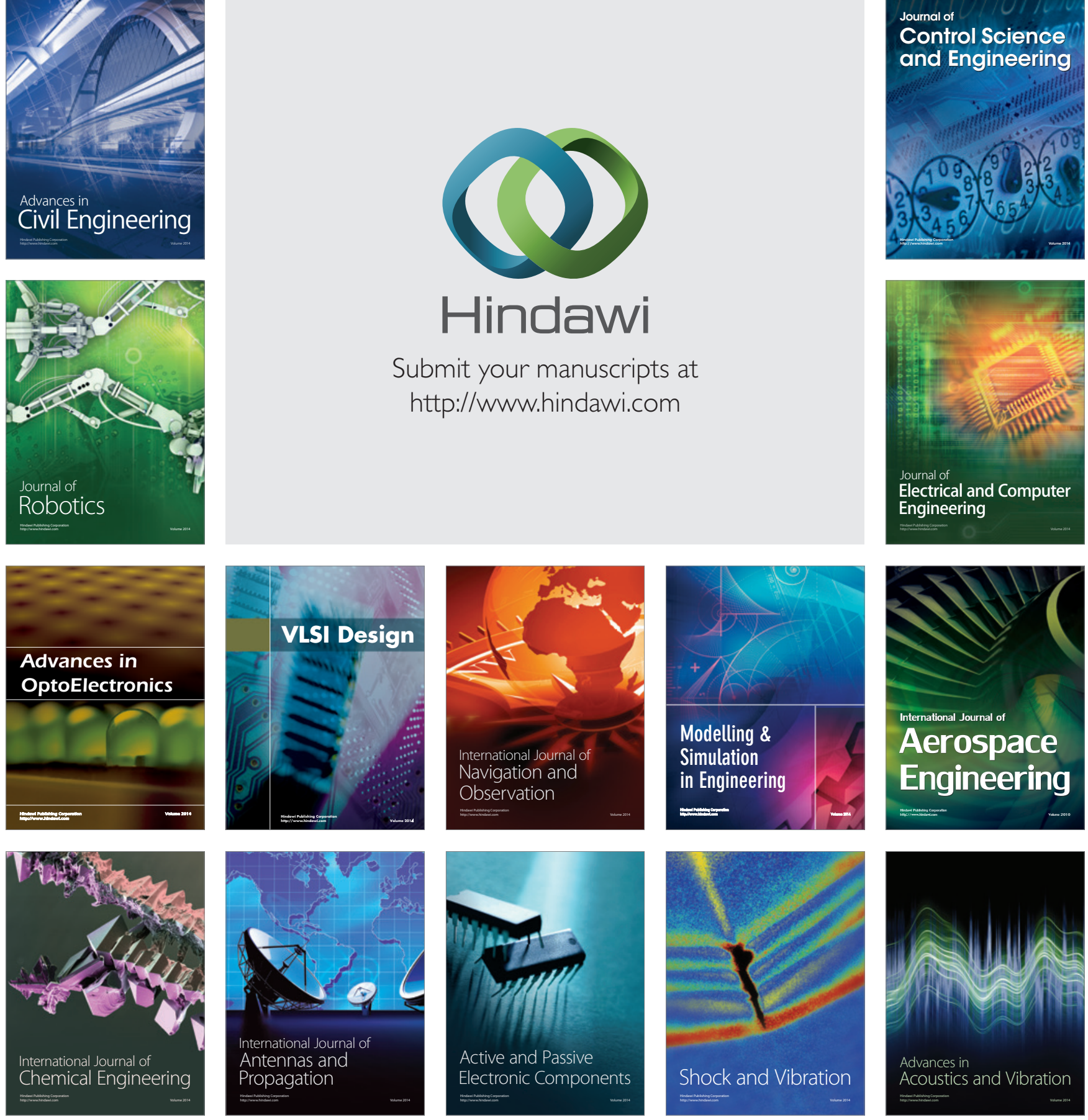